\title{
NIVELES DE COMPETENCIAS ACADÉMICAS Y EL DESEMPEÑO EN LA PRÁCTICA PREPROFESIONAL DE ESTUDIANTES DE LA ESCUELA DE CONTABILIDAD DE LA UNIVERSIDAD CÉSAR VALLEJO 2015 Levels of academic competences and performance in the pre-professional practice of students of the Accounting School of César Vallejo University 2015
}

\author{
Raúl Delgado ${ }^{1}$, Rey León ${ }^{2,3}$, María Torrelio ${ }^{3}$, Richard Cisneros ${ }^{2,3}$, Luis Aguilar $^{2,3}$
}

\begin{abstract}
Resumen
El presente artículo tiene como objetivo determinar la relación entre los niveles de competencias académicas y el desempeño en la práctica preprofesional de estudiantes de la Escuela de Contabilidad. Es un estudio correlacional de diseño no experimental transversal; se trabajó con una población total de 60 estudiantes, se aplicó dos instrumentos denominados cuestionario sobre niveles de competencias académicas y cuestionario sobre el desempeño en la práctica preprofesional, uno a los estudiantes y el otro a los jefes directos de los estudiantes. Se determinó una relación positiva y significativa entre los niveles de competencias académicas y el desempeño en la práctica preprofesional.
\end{abstract}

Palabras clave: competencias académicas, desempeño, práctica preprofesional

\begin{abstract}
This study aims to determine the relationship between levels of academic skills and performance in preprofessional practice of students from the accounting school. This is a cross-correlational not experimental design based on the study of 60 students, which consisted on the application of two instruments, one for students and the other was applied to their direct managers. The results of this research show that there is a positive and significant relation between the levels of academic skills and the performance of preprofessional practice.
\end{abstract}

Keywords: academic skill, performance, preprofessional practice

1 Universidad César Vallejo

2 Universidad Peruana Cayetano Heredia

3 Universidad Femenina del Sagrado Corazón

rey.leon@neurocienciaperu.org 


\section{INTRODUCCIÓN}

Actualmente nos encontramos en un mundo bastante competitivo, donde el mercado laboral es cada día más exigente; de las instituciones universitarias egresan profesionales que ingresan al mercado laboral pero no todos cuentan con un nivel académico que permita un adecuado desempeño laboral.

Es necesario y urgente que los alumnos de los últimos ciclos se encuentren con un adecuado nivel de competencias, por ello es necesario medir sus destrezas y detectar las deficiencias que puedan tener para presentar alternativas de solución durante su formación académica.

\section{ANTECEDENTES}

Las variables de esta investigación son Niveles de competencias académicas y el Desempeño en las prácticas preprofesionales, las cuales han sido analizadas a través de sus dimensiones: conocimientos, destreza y actitudes.

Charria, Sarsosa, Uribe, López y Arenas (2011), citando a la comisión de la Secretary's Comisión on Achieving Necessary Skills (SCANS), afirman que las competencias académicas "están asociadas a conocimientos fundamentales que se adquieren en la formación general" (p. 141), y las clasifican en habilidades básicas: capacidad lectora, escritura, matemáticas, hablar y escuchar; desarrollo de pensamiento, constituido por pensamiento creativo, solución de problemas, toma de decisiones, capacidad de aprender y razonar, asimilación, comprensión; y en cuánto al desempeño en la práctica preprofesional incorpora las siguientes cualidades personales: la autorresponsabilidad, autoestima, sociabilidad, autodirección e integridad.

Existe variedad en la descripción del término competencia; autores como Mertens (2000), Vargas (2004), Romero (2005), establecen en sus definiciones distintas orientaciones como: empresarial, educativo, psicológico. Sin embargo, se puede definir competencia desde el punto de vista de la personalidad. Martha Alles (2006, 2007 y 2008) hace referencia a características de la personalidad, que generan un desempeño exitoso en un puesto de trabajo. Cada puesto de trabajo puede tener diferentes características en las empresas y /o mercados distintos, pudiendo englobar varios conceptos, desde los conocimientos hasta las competencias propiamente dichas.

\section{PROBLEMA}

La manera de expresar un problema varía según la opinión de diversos autores; en el desarrollo de esta investigación se tomará en cuenta la propuesta de Kerlinger (2008).

\section{Problema general:}

¿Qué relación existe entre los niveles de competencias académicas y el desempeño en las prácticas preprofesionales de los estudiantes del IX ciclo de la Escuela de Contabilidad de la Universidad César Vallejo 2015?

\section{Problemas específicos:}

¿Qué relación existe entre los niveles de competencias académicas con la dimensión de conocimientos y el desempeño en las prácticas preprofesionales de los estudiantes del IX ciclo de la Escuela de Contabilidad de la Universidad César Vallejo 2015?

¿Qué relación existe entre los niveles de competencias académicas con la dimensión destreza y el desempeño en las prácticas preprofesionales de los estudiantes del IX ciclo de la Escuela de Contabilidad de la Universidad César Vallejo 2015?

¿Qué relación existe entre los niveles de competencias académicas con la dimensión actitudes y el desempeño en las prácticas preprofesionales de los estudiantes del IX ciclos de la Escuela de Contabilidad de la Universidad César Vallejo?

\section{OBJETIVOS}

\section{Objetivo general}

Determinar la relación que existe entre los niveles de competencias académicas y el desempeño en las prácticas preprofesionales de los estudiantes del IX ciclo de la Escuela de Contabilidad de la Universidad César Vallejo 2015.

\section{Objetivos específicos}

Determinar la relación que existe entre los niveles de competencias académicas con la dimensión 
conocimientos y el desempeño en las prácticas preprofesionales de los estudiantes del IX ciclo de la Escuela de Contabilidad de la Universidad César Vallejo 2015.

Determinar la relación que existe entre los niveles de competencias académicas con la dimensión destreza y el desempeño en las prácticas preprofesionales de los estudiantes del IX ciclo de la Escuela de Contabilidad de la Universidad César Vallejo 2015.

Determinar la relación que existe entre los niveles de competencias académicas con la dimensión actitudes y el desempeño en las prácticas preprofesionales de los estudiantes del IX ciclo de la Escuela de Contabilidad de la Universidad César Vallejo 2015.

\section{METODOLOGÍA}

Se utilizó el método hipotético deductivo. Según Hernández, Fernández y Baptista (2014) esta propuesta considera que el tipo de razonamiento "comienza con la teoría derivándose expresiones lógicas denominadas hipótesis que el investigador busca someter a prueba" (p. 6). De acuerdo con los autores, "las hipótesis se contrastan con la realidad para aceptarse o rechazarse en un contexto determinado" (p. 114).

Siguiendo a Sánchez y Reyes (1996), el presente trabajo de investigación es de tipo básica y sustantiva. Es básica porque está orientada a la búsqueda de nuevos conocimientos y nuevos campos de investigación. Además, recoge información de la realidad para enriquecer el conocimiento científico, orientándose al descubrimiento de principios y leyes. Es sustantiva porque trata de responder a los problemas teóricos o sustantivos; en tal sentido, está orientada, a describir, explicar, predecir la realidad, con lo cual se va en búsqueda de principios y leyes generales que permita organizar una teoría científica.

Según Hernández et al. (2014), el nivel es descriptivo correlacional. Los estudios correlacionales "asocian variables mediante un patrón predecible para un grupo o población". (p. 93). Además, tienen "como finalidad conocer la relación o grado de asociación que exista entre dos o más conceptos, categorías o variables en un contexto en particular" (p. 93). Por lo tanto, en este estudio se trata de determinar la relación sobre el estado actual del fenómeno, esto es asociar el nivel de competencias académicas y el desempeño en la práctica preprofesional del estudiante del IX ciclo de la Escuela de Contabilidad de la Universidad César Vallejo 2015. Según Hernández et al. (2014, p. 154), es un diseño no experimental porque se realiza "sin la manipulación deliberada de variables; y en los que solo se observan los fenómenos en su ambiente natural" para después realizar el análisis en su forma de investigación transeccional correlacional.

ParaHernándezetal.(2014,p.157)estranseccional correlacional porque "describen relaciones entre dos o más categorías, conceptos, o variables en un momento determinado". El gráfico que corresponde a este diseño es el siguiente:

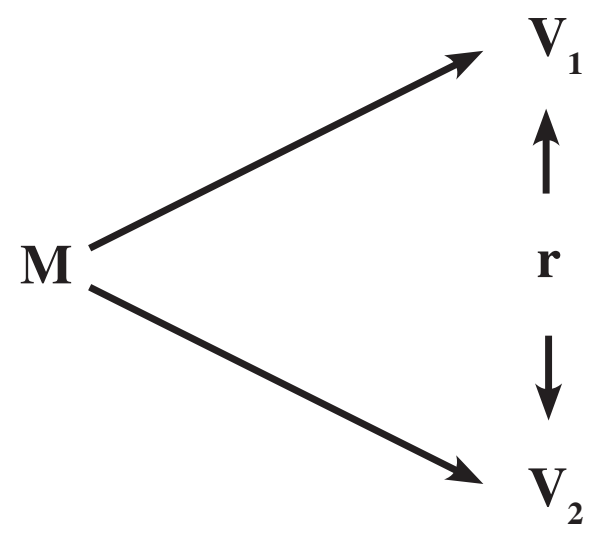

Donde:

$\mathrm{M} \rightarrow$ Representa a los 60 estudiantes del IX ciclo de la Escuela de Contabilidad de la Universidad César Vallejo 2015

V1 $\rightarrow$ Representa el nivel de competencia académica.

V2 $\rightarrow$ Representa el desempeño en la práctica preprofesional en el área contable.

$r \rightarrow$ Representa la relación que existe entre el nivel de competencias académicas y el desempeño en la práctica preprofesional en la Escuela de Contabilidad de los estudiantes del IX ciclo de la Universidad César Vallejo2015.

Hernández et al. (2014, p. 174), citando a Lepkowski, definen a la población como "el conjunto de todos los casos que concuerdan con una serie 
de especificaciones". El marco poblacional está constituido por 60 estudiantes del IX ciclo de la Escuela de Contabilidad, de la Universidad César Vallejo 2015. La población es censal.

\section{Instrumentos}

En este estudio se usó la técnica de la encuesta, de acuerdo con Hernández et al. (2014). La encuesta es el procedimiento adecuado para recolectar datos a grandes muestras en un solo momento. En este estudio se asume dicha técnica, para trabajar con la población censal de los estudiantes del IX ciclo de la Escuela de Contabilidad de la Universidad César Vallejo 2015.

Se empleó el instrumento denominado cuestionario aplicado a los estudiantes para evaluar sus niveles de competencias académicas y otro a los Jefes de los estudiantes que evaluaron su desempeño en la práctica preprofesional.

En el estudio de la investigación se utilizó las siguientes técnicas:

Técnica de análisis documental: para la elaboración de los instrumentos se tomaron como referencia los instrumentos de las diversas tesis consultadas en los antecedentes, como Boude (2011), Andrade (2013), Barranco (2004), Alsina et al. (2011), entre otros.

Técnica de la encuesta: bajo esta modalidad se aplicó un cuestionario con preguntas directas con alternativas múltiples, teniendo como base la escala de Likert.

Técnica de juicio de expertos: esta técnica sirvió para validar el instrumento de recolección de datos de los estudiantes participantes de la investigación a juicio de expertos, siendo cuatro especialistas de la escuela con experiencia en investigación del problema propuesto y docentes en actividad. La validez de expertos "se refiere al grado en que aparentemente un instrumento de medición mide la variable en cuestión, de acuerdo a 'voces calificadas' " Hernández et al. (2014, p. 204).

El trabajo de investigación consta de dos cuestionarios denominados cuestionario sobre niveles de competencias académicas y cuestionario sobre el desempeño en la práctica preprofesional, los cuales permitieron recolectar datos cuantitativos de nuestras variables.

\section{RESULTADOS}

Tabla 1.

Niveles de competencias académicas * Desempeño en la práctica preprofesional

\begin{tabular}{|c|c|c|c|c|c|}
\hline & & & \multicolumn{3}{|c|}{$\begin{array}{c}\text { Desempeño en la práctica } \\
\text { preprofesional }\end{array}$} \\
\hline & & & Malo & Bueno & Total \\
\hline \multirow{4}{*}{$\begin{array}{l}\text { Niveles de } \\
\text { competencias } \\
\text { académicas }\end{array}$} & & Recuento & 27 & 5 & 32 \\
\hline & Bajo & $\%$ del total & $45,00 \%$ & $8,33 \%$ & $53,33 \%$ \\
\hline & & Recuento & 7 & 21 & 28 \\
\hline & Alto & $\%$ del total & $11,67 \%$ & $35,00 \%$ & $46,67 \%$ \\
\hline \multirow[t]{2}{*}{ Total } & & Recuento & 34 & 26 & 60 \\
\hline & & $\%$ del total & $56,67 \%$ & $43,33 \%$ & $100,0 \%$ \\
\hline
\end{tabular}

Fuente: Creación personal

En cuanto a descripción de resultados: Se observó que existe un grupo representativo del 45,0\% que tiene un desempeño malo en la práctica preprofesional y presenta niveles de competencia académica bajos, mientras que el 35,0\% tiene un desempeño bueno en la práctica preprofesional y niveles de competencia académica altos (ver tabla 1).

Tabla 2

Niveles de competencias académicas y los conocimientos

\begin{tabular}{|c|c|c|c|c|c|}
\hline & & & \multicolumn{3}{|c|}{$\overline{\text { Conocimientos }}$} \\
\hline & & & Malo & Bueno & Total \\
\hline \multirow{4}{*}{$\begin{array}{l}\text { Niveles de } \\
\text { competencias } \\
\text { académicas }\end{array}$} & & Recuento & 29 & 3 & 32 \\
\hline & Bajo & $\%$ del total & $48,33 \%$ & $5,00 \%$ & $53,33 \%$ \\
\hline & & Recuento & 17 & 11 & 28 \\
\hline & Alto & $\%$ del total & $28,33 \%$ & $18,33 \%$ & $46,66 \%$ \\
\hline \multirow[t]{2}{*}{ Total } & & Recuento & 46 & 14 & 60 \\
\hline & & $\%$ del total & $76,66 \%$ & $23,33 \%$ & $100,0 \%$ \\
\hline
\end{tabular}

Fuente: Creación personal

Se observó que los estudiantes que representan el $48,3 \%$ con niveles de competencias académicas bajos, 
presentan un nivel malo en conocimientos, y el 18,3\% de estudiantes tiene niveles altos de competencias académicas y un buen conocimiento en el desempeño de la práctica preprofesional (ver tabla 2).

Tabla 3

Niveles de competencias académicas y las destrezas

\begin{tabular}{|c|c|c|c|c|c|}
\hline & & & & & Destrezas \\
\hline & & & Malo & Bueno & Total \\
\hline & & Recuento & 31 & 1 & 32 \\
\hline Niveles de & Bajo & $\%$ del total & $51,67 \%$ & $1,67 \%$ & $53,34 \%$ \\
\hline $\begin{array}{l}\text { competencias } \\
\text { académicas }\end{array}$ & Alto & $\begin{array}{l}\text { Recuento } \\
\% \text { del total }\end{array}$ & $\begin{array}{c}17 \\
28,33 \%\end{array}$ & $\begin{array}{c}11 \\
18,33 \%\end{array}$ & $\begin{array}{r}28 \\
46,66 \%\end{array}$ \\
\hline Total & & $\begin{array}{l}\text { Recuento } \\
\% \text { del total }\end{array}$ & $\begin{array}{c}48 \\
80,00 \%\end{array}$ & $\begin{array}{c}12 \\
20,00 \%\end{array}$ & $\begin{array}{r}60 \\
100,0 \%\end{array}$ \\
\hline
\end{tabular}

Fuente: Creación personal

Se observó que los estudiantes que representan el $51,7 \%$ con niveles de competencias académicas bajos, presentan un nivel malo con los conocimientos, mientras que el 18,3\% de estudiantes tienen niveles altos de competencias académicas y un buen nivel de destrezas en el desempeño de la práctica preprofesional (ver tabla 3).

Tabla 4

Niveles de competencias académicas y las actitudes

\begin{tabular}{|c|c|c|c|c|c|}
\hline & & & \multicolumn{3}{|c|}{ Actitud } \\
\hline & & & Malo & Bueno & Total \\
\hline \multirow{3}{*}{$\begin{array}{l}\text { Niveles de } \\
\text { competencias } \\
\text { académicas }\end{array}$} & & Recuento & 28 & 4 & 32 \\
\hline & Bajo & $\%$ del total & $46,67 \%$ & $6,67 \%$ & $53,34 \%$ \\
\hline & Alto & $\begin{array}{l}\text { Recuento } \\
\% \text { del total }\end{array}$ & $\begin{array}{c}4 \\
6,67 \%\end{array}$ & $\begin{array}{c}24 \\
40,00 \%\end{array}$ & $\begin{array}{c}28 \\
46,67 \%\end{array}$ \\
\hline Total & & $\begin{array}{l}\text { Recuento } \\
\% \text { del total }\end{array}$ & $\begin{array}{c}32 \\
53,34 \%\end{array}$ & $\begin{array}{c}28 \\
46,67 \%\end{array}$ & $\begin{array}{c}60 \\
100,0 \%\end{array}$ \\
\hline
\end{tabular}

Fuente: Creación personal

Se observó en los estudiantes con niveles de competencias académicas bajos, que el $46,7 \%$ presenta un nivel malo en actitud, mientras que el 40,0\% de estudiantes tiene niveles altos de competencias académicas y buenas actitudes en el desempeño de la práctica preprofesional (ver tabla 4).

En cuanto a las hipótesis se concluyó lo siguiente: Que el coeficiente de correlación de Rho de Spearman, tomando como criterio de aceptación correlaciones con significancia al $\mathrm{p}<0.05$, nos indica que existe una relación positiva y moderada significativa entre los niveles de competencias académicas y el desempeño en la práctica preprofesional $(\mathrm{r}=0,598, \mathrm{p}=0,000)$. Por lo tanto, se acepta; existe relación positiva y significativa entre los niveles de competencias académicas y el desempeño en la práctica preprofesional de los estudiantes.

Asimismo, nos indica que existe una relación positiva significativa entre los niveles de competencias académicas y los conocimientos $(\mathrm{r}=0,353, \mathrm{p}=0,006)$. Por lo tanto, existe relación positiva y significativa entre los niveles de competencias académicas y la dimensión conocimientos en el desempeño en la práctica preprofesional de los estudiantes

Se observó también que existe relación positiva y significativa entre los niveles de competencias académicas y las destrezas $(\mathrm{r}=0,451, \mathrm{p}=, 000)$. Por lo tanto, existe relación positiva y significativa entre los niveles de competencias académicas y la dimensión destrezas en el desempeño en la práctica preprofesional de los estudiantes.

Se observó como criterio de aceptación, correlaciones con significancia al $\mathrm{p}<0.05$. se concluye que existe una relación positiva y altamente significativa entre los niveles de competencias académicas y las actitudes $(\mathrm{r}=0,732, \mathrm{p}=, 000)$. Por lo tanto, existe relación positiva y significativa entre los niveles de competencias académicas y la dimensión actitudes en el desempeño en la práctica preprofesional de los estudiantes.

\section{DISCUSIÓN}

Aunque el resultado no fue altamente significativo, existe coincidencia con los autores que se presentó en los antecedentes, Charria et al. (2011). De los resultados se observa que existe relación entre las dos variables propuestas - niveles de competencias académicas y desempeño en las prácticas preprofesionales-, tanto a nivel general como específico con cada una de las 
dimensiones propuestas; por lo tanto, se debe de hacer una revisión del plan curricular para adecuarlo a las necesidades que exige el mercado laboral.

Finalmente, esta investigación brinda aporte significativo a futuras investigaciones, nuevas estrategias y métodos para desarrollar un adecuado seguimiento a los niveles de competencias académicas con el objetivo de lograr un adecuado desempeño en la práctica preprofesional de los estudiantes.

\section{CONCLUSIONES}

Este estudio ha sido de gran ayuda para poder evaluar los niveles de competencias académicas que tienen los discentes de los últimos ciclos y cómo se encuentran preparados para enfrentarse al campo laboral. De acuerdo con los resultados obtenidos se determina que existe relación entre ambas variables estudiadas. Así, si se presentan niveles de competencias académicas bajos, el desempeño será malo en las prácticas preprofesionales.

Se requiere hacer un estudio del plan curricular para mejorarlo y actualizarlo, que esté acorde con los tiempos en que vivimos, y los estudiantes demuestren competencias que les permita desempeñarse con eficiencia en el campo laboral.

\section{RECOMENDACIONES}

1) Analizar detenidamente el plan curricular de la Escuela de Contabilidad y adecuarla a las necesidades actuales y requerimientos que todo profesional contable necesita conocer que le permita desempeñarse adecuadamente en el campo contable. Porque en el campo laboral las empresas realizan evaluaciones por competencias y no solamente académicas, en consecuencia, deberá hacerse un estudio pormenorizado del currículo y enfocarlo hacia la cultura de la empleabilidad de los estudiantes y la misma sea basada en competencias.

2) Serecomiendaqueseimplementenlaboratorios en la Escuela de Contabilidad con programas o software contables, ya que esta carece de laboratorios exclusivos para su enseñanza, que permita a los discentes lograr desarrollar competencias académicas y laborales. En estos tiempos toda la información se lleva con formato electrónico (empresas, SUNAT, como los comprobantes, libros electrónicos, PDT, etc.). Urge implementar con urgencia en la malla curricular.

3) Aplicar modelos de enseñanza centrado en el estudiante que incluyan estrategias y métodos de aprendizaje donde se les enseñe a analizar los problemas, con simulaciones a la realidad laboral, que el profesional contable no se circunscriba a un modelo técnico como preparador de información y asuma modelos de liderazgo con la preparación adecuada (asesor tributario $\mathrm{y} / \mathrm{o}$ financiero).

4) El presente trabajo pone de manifiesto la importancia de desarrollar valores laborales como la eficiencia, la inteligencia personal, la creatividad, la puntualidad, la disciplina, la proactividad que permita demostrar siempre una buena actitud en su centro de labores.

5) Finalmente se concluye que se debe fomentar la cultura de un proceso de mejora continua adaptable a los tiempos de manera dinámica, (tecnología y estrategias de aprendizaje) en los futuros profesionales conforme a la demanda del mercado.

\section{REFERENCIAS}

Alles, M. (2006). Influencia de las características de personalidad (competencias) en la empleabilidad de profesionales. Buenos Aires: Universidad de Buenos Aires. Facultad de Ciencias Económicas. Recuperado de http://bibliotecadigital.econ.uba. ar/download/tesis/1501-1181_AllesMA.pdf

Alles, M. (2007). Desarrollo del talento humano basado en competencias. Buenos Aires, México, Santiago, Montevideo: Ediciones Granica, S.A. Recuperado de http://saludpublica.bvsp.org.bo/ textocompleto/bvsp/boxp68/talento-humano_ a.pdf

Alles, M. (2008). Dirección estratégica de Recursos Humanos: Gestión por competencias. Buenos Aires, México, Santiago, Montevideo: Ediciones Granica, S.A. Recuperado de https:// jgestiondeltalentohumano.files.wordpress. com/2013/11/direccic3b3n-estratc3a9gica- 
de-recursos-humanos-gestic 3 b $3 n$-porcompetencias-casos-martha-alles.pdf

Alsina, J., Boix R., Burset, S., Buscá, F. Colomina, R., García, M., Mauri, T., Pujolá, J. y Sayós, R. (2011). Evaluación por competencias en la universidad: Las competencias transversales. Barcelona: Ediciones Octaedro. Recuperado de http://www.ub.edu/ice/sites/default/files/docs/ qdu/18cuaderno.pdf

Andrade, J. (2013). Prácticas pre-profesionales para el desarrollo de competencias de los estudiantes de psicología educativa de la Universidad laica Vicente Rocafuerte para la optimización de su formación y propuesta de un sistema alternativo (Tesis de Maestría, Universidad de Guayaquil, Guayaquil, Ecuador). Recuperada de http:// repositorio.ug.edu.ec/bitstream/redug/1701/1/Fin al\%20Tesis\%20de\%20J\%C3\%A9ssica\%20Andr ade\%20total1234jl.pdf

Barranco, L. (2004). Propuesta metodológica para la práctica pre-profesional en la asignatura de práctica forense ministerial de la licenciatura en Derecho de la Universidad Autónoma del Estado de Hidalgo (Tesis de Maestría, Universidad Autónoma del Estado de Hidalgo, Pachuca de Soto, Hidalgo, México). Recuperada de http://www.uaeh.edu.mx/docencia/Tesis/icshu/ maestria/documentos/Propuesta\%20dmetodologi ca\%20para\%20la\%20practica.pdf

Boude, O. (2011). Desarrollo de competencias genéricas y específicas en educación superior a través de una estrategia didáctica medida por TIC (Tesis de Maestría, Universidad Nacional de Educación a Distancia, Madrid). Recuperada de http://e-spacio.uned.es/fez/eserv/tesisuned: Educacion-Orboude/Documento.pdf

Charria, V., Sarsosa, K., Uribe, A., López, C., Arenas, F. (2011). Definición y clasificación teórica de las competencias académicas, profesionales y laborales. Las competencias del psicólogo en Colombia. Psicología desde el Caribe, 28, 133-165 (Universidad del Norte, Barranquilla, Colombia). Recuperado de http://www.redalyc. org/pdf/213/21320758007.pdf

Hernández, R., Fernández, C. y Baptista, M. P. (2014). Metodología de la investigación $\left(6^{\mathrm{a}}\right.$ ed.). México: Mc Graw-Hill. Recuperado de https://metodologiaaecs.wordpress.com/2016/ 01/31 libro-metodologia-de-la-investigacion-6taedicion-sampieri-pdf/

Kerlinger,F.(2008).Investigación delcomportamiento. México: McGraw-Hill.

Mertens, L. (2000). La gestión por competencia en la empresa y la formación profesional. Madrid, España: Ediciones de la Organización de Estados Iberoamericanos para la Educación, la Ciencia y la Cultura (OEI), Editorial Bravo Murillo. Recuperado de http://www.oei.es/historico/ oeivirt/fp/iberfop01.htm

Romero, M. (2005). ¿Y qué son las competencias? ¿Quién las construye? ¿Por qué competencias? Revista Electrónica Educar, 35, 9-18. Recuperado de http://www.quadernsdigitals.net/datos/ hemeroteca/r_24/nr_729/a_9909/9909.pdf

Sánchez, H. y Reyes, C. (1996). Metodología y diseños en la investigación científica: aplicados a la psicología, educación y ciencias sociales $\left(2^{\mathrm{a}}\right.$ ed.). Lima: Mantaro.

Vargas. F. (2004). Las 40 preguntas más frecuentes en competencia laboral. Cinterfor/OIT Recuperado de https://www.oitcinterfor.org/ publicaci\%C3\%B3n/40-preguntas-sobrecompetencia-laboral

Fecha de recepción: 29 de noviembre, 2016

Fecha de aceptación: 6 de diciembre, 2016 